\title{
Deformation Quantization of Lagrangian Fiber Bundles
}

\author{
Nicolai Reshetikhin and Milen Yakimov \\ Department of Mathematics, \\ University of California at Berkeley, \\ Berkeley, CA 94720, USA* \\ Dedicated to the memory of Moshé Flato
}

\begin{abstract}
Let $(M, \omega)$ be a symplectic manifold. A Lagrangian fiber bundle $\pi: M \rightarrow B$ determines a completely integrable system on $M$. First integrals of this system are the pull-backs of functions on the base of the bundle. We show that for each Lagrangian fiber bundle $\pi$ there exist star products on $C^{\infty}(M)[[\hbar]]$ which do not deform the pointwise multiplication on the subalgebra $\pi^{*}\left(C^{\infty}(B)\right)[[\hbar]]$. The set of equivalence classes of such star products is in bijection with formal deformations of the symplectic structure $\omega$ for which $\pi: M \rightarrow B$ remains Lagrangian taken modulo formal symplectomorphisms of $M$.
\end{abstract}

\section{Introduction}

The phase space of a classical Hamiltonian system is a symplectic manifold $(M, \omega)$. Smooth functions on the phase space form classical algebra of observables. It is a Poisson algebra with Lie bracket given by

$$
\{f, g\}=\omega^{-1}(d f \wedge d g), \quad f, g \in C^{\infty}(M)
$$

( $\omega^{-1} \in \wedge^{2} T M$ is the bivector field dual to the two-form $\omega$ ) and commutative product the pointwise product on $C^{\infty}(M)$.

A completely integrable classical Hamiltonian system on a $2 n$-dimensional symplectic manifold admits $n$ independent integrals which commute with respect to the Poisson bracket. Geometrically this means that away from the singular level surfaces the symplectic manifold $M$ is fibered over an open subset of $\mathbb{R}^{n}$. More generally for any fiber bundle $\pi: M \rightarrow B$, whose fibers are Lagrangian submanifolds of $(M, \omega)$ the subalgebra $\pi^{*}\left(C^{\infty}(B)\right)$ of $C^{\infty}(M)$ is Poisson commutative. This a geometric model for a completely integrable classical system without singularities.

Quantization as a way to pass from classical Hamiltonian mechanics to quantum mechanics mathematically can be regarded as a procedure of replacing the classical

*E-mail addresses: reshetik@math.berkeley.edu, yakimov@math.berkeley.edu 
algebra of observables (the Poisson algebra of functions on a symplectic manifold) by an associative noncommutative algebra of quantum observables. One approach to quantization is to consider the algebra of quantum observables as a (formal) deformation of the Poisson algebra $\left(C^{\infty}(M),\{.,\}.\right)$ of classical observables in the category of associative algebras. For precise definitions see Sect. 2. This approach is known as deformation quantization. It was pioneered by H. Weyl [18], and J. Moyal [14], who considered the case of symplectic vector spaces and developed further by J. Vey [16], F. Berezin [2] and F. Bayen, M. Flato, C. Fronsdal, A. Lichnerowicz, and D. Sternheimer [3] in the case of general Poisson manifolds. There were several techniques employed in the study of the deformations of the Poisson algebra $\left(C^{\infty}(M),\{.,\}.\right)$. In [16, 3] a cohomological technique, based on results of [10] was developed. It was successively used in [6] to prove that any symplectic manifold can be quantized. Fedosov [8] found a geometric construction of deformations in the symplectic case. Finally the problem of quantization was completely solved by Kontsevich [12] for any Poisson manifold. He derived it from the more general result of formality of the differential graded Lie algebra of the Hochschild complex of functions on any smooth manifold.

For a Lagrangian fiber bundle $\pi: M \rightarrow B$ inside the algebra of classical observables $C^{\infty}(M)$ sits the Poisson commutative subalgebra of classical integrals of motion $\pi^{*}\left(C^{\infty}(B)\right)$. It is natural to ask two questions in this situation:

1. Is it possible to construct a quantization of the Poisson algebra $\left(C^{\infty}(M),\{.,\}.\right)$ such that the subalgebra $\pi^{*}\left(C^{\infty}(B)\right)$ will not deform and thus will form an algebra of quantum integrals of motion?

2. If it is possible, then "how many of such quantizations" one can construct?

In this paper we give answers to both questions using Fedosov's technique. Our results are summarized in the following Theorem.

Theorem 1.1 ( $i)$ The classes of star products on $(M, \omega)$ which are equivalent to products keeping the commutative algebra $\pi^{*}\left(C^{\infty}(B)\right)$ undeformed are in one to one correspondence with the deformations of the two-form $\omega$ in $H^{2}(M)[[\hbar]]$ having representatives in $Z^{2}(M)[[\hbar]]$ with respect to which the bundle $\pi: M \rightarrow B$ remains Lagrangian.

(ii) Any star product on $(M, \omega)$ for which the space $\pi^{*}\left(C^{\infty}(B)\right)[[\hbar]]$ generates a commutative subalgebra of $C^{\infty}(M)[[\hbar]]$ is equivalent to one that keeps the usual product on $\pi^{*}\left(C^{\infty}(B)\right)[[\hbar]]$ undeformed.

This Theorem provides a positive answer to Question 1. In the framework of Fedosov's quantization the formal two-form associated to a class of star products on $(M, \omega)$ with the decsribed properties is its characteristic form (class). In view of [13], the discussed classes of star products on $(M, \omega)$ are in one to one correspondence with formal $(\mathbb{R}[[\hbar]])$ deformations of the symplectic structure $\omega$ on $M$ for which $\pi: M \rightarrow B$ remains Lagrangian, taken modulo the action of the group of formal symplectomorphisms of $(M, \omega)$ starting with the identity diffeomorphism of $M$. 
Remark 1.2 Often a quantization $\star$ of $(M, \omega)$ is apriori given (e.g. quantization of a cotangent bundle in terms of differential operators). In this situation one wants to deform (if it is possible) the Poisson commutative algebra $\pi^{*}\left(C^{\infty}(B)\right)$ to a commutative subalgebra of $(C M[[\hbar]], \star)$ by introducing quantum corrections to the commuting quantities. For this problem Theorem 1.1 can be used in the following manner. The first step is to check whether the characteristic form of the product $\star$ is of the required type. If it is so, let $*$ be an equivalent to $\star$ product which keeps the algebra $\pi^{*}\left(C^{\infty}(B)\right)$ undeformed and the corresponding formal equivalence be given by the differential operator $P=\mathrm{id}+O(\hbar)$. (An iterative procedure for constructing such $*$ is contained in Sect. 5.) Then $\left(P^{-1}\left(\pi^{*}\left(C^{\infty}(B)\right)\right)[[\hbar]], \star\right)$ is a commutative subalgebra of $\left(C^{\infty}(M)[[\hbar]], \star\right)$ and the quantum correction to each classical integral of motion $f \in \pi^{*}\left(C^{\infty}(B)\right)$ is $\left(P^{-1}-\mathrm{id}\right)(f)$.

The paper is organized as follows. In Sect. 2 we remind some basic definitions and facts about Fedosov's quantization. Sect. 3 contains the construction of special connections which we use in Sections 4 and 5. The proof of the existence of associative star products on $C^{\infty}(M)[[\hbar]]$ preserving given Lagrangian fibration is presented in Sect. 4. Sect. 5 is devoted to the classification of such star products. Theorem 1.1 is a combination of Theorem 4.1 and Theorem 5.1.

One can restrict to the set of star products on $(M, \omega)$ that keep $\pi^{*}\left(C^{\infty}(B)\right)[[\hbar]]$ undeformed and consider a finer equivalence relation given by differential operators that preserve $\pi^{*}\left(C^{\infty}(B)\right)[[\hbar]]$. A classification result in this setting as well as extensions to the case of noncommutative integrable systems will be discussed in a separate publication.

\section{Acknowledgements}

We benefited from discussions with B. Fedosov, A. Givental, L. Takhtajan, and A. Weinstein whom we thank for this. We thank also Technishe Universität Berlin for the warm hospitality when this work was being completed. The research of N.R. was partially supported by NSF grant DMS96-03239, and M.Y. received support from NSF grants DMS96-03239 and DMS94-00097.

\section{Deformation Quantization and Fedosov's Construc- tion}

\subsection{Basic Notions}

Definition 2.1 A formal local star product on a Poisson manifold with Poisson bracket $\{.,$.$\} is an associative \mathbb{R}[[\hbar]]$-linear product on $C^{\infty}(M)[[\hbar]]$ of the type

$$
f * g=f g+\sum_{l=1}^{\infty} \hbar^{l} Q_{l}(f, g), \quad f, g \in C^{\infty}(M)[[\hbar]],
$$

where

1. $Q_{l}$ are local (i.e. bidifferential) operators, 
2. $f * g-g * f=\hbar\{f, g\}+O\left(\hbar^{2}\right), \forall f, g \in C^{\infty}(M)[[\hbar]]$,

3. $1 * f=f * 1=f$, i.e $Q_{l}(1, f)=Q_{l}(f, 1)=0, \forall f \in C^{\infty}(M)[[\hbar]], l \geq 1$.

In this paper we will consider only formal local star products and we will call them just star products.

Definition 2.2 Two star products $*_{1}$ and $*_{2}$ are called equivalent if there exists a local (i.e. differential) $\mathbb{R}[[\hbar]]$-linear operator $P: C^{\infty}(M)[[\hbar]] \rightarrow C^{\infty}(M)[[\hbar]]$ of the type

$$
P=\mathrm{id}+\sum_{l=1}^{\infty} \hbar^{l} P_{l}
$$

for some differential operators $P_{l}$ on $M$ such that

$$
P\left(f *_{1} g\right)=P f *_{2} P g, \quad \forall f, g \in C^{\infty}(M) .
$$

For more details on these definitions we refer to [3, 14].

\subsection{The Weyl Bundle}

Let $(M, \omega)$ be a symplectic manifold of dimension $2 n$. The Weyl bundle $W$ on $M$ is defined by

$$
W=S\left(T^{*} M\right)
$$

where $S(V)$ denotes the completed symmetric power $S(V)=\coprod_{l=0}^{\infty} S^{l}(V)$ of a vector space $V$. For any point $x \in M$ the commutative algebra $S\left(T_{x}^{*} M\right)$ can be naturally identified with the algebra $\mathcal{J}_{0}^{\infty}\left(T_{x} M\right)$ of $\infty$-jets of real valued functions on $T_{x} M$ at 0 . More often we will use the bundle

$$
W_{\hbar}=S\left(T^{*} M\right)[[\hbar]]=W[[\hbar]],
$$

where $\hbar$ is a formal variable and will still call it Weyl bundle of $M$.

The tangent space $T_{x} M$ at any $x \in M$ is a symplectic vector space and the MoyalWeyl product equips any fiber $\left(W_{\hbar}\right)_{x}$ of $W_{\hbar}$ with a structure of noncommutative associative algebra. It becomes a graded algebra if we put

$$
\begin{aligned}
& \operatorname{deg} v=l, \quad \text { for } v \in S^{l}\left(T_{x}^{*} M\right) \\
& \operatorname{deg} \hbar=2 .
\end{aligned}
$$

The homogeneous component of $\left(W_{\hbar}\right)_{x}$ in degree $l$ will be denoted by $\left(W_{\hbar}^{l}\right)_{x}$. The fiberwise product on $W_{\hbar}$ determines an associative product on the space $\Gamma\left(W_{\hbar}\right)$ of global sections of $W_{\hbar}$, denoted by $\circ$.

In local coordinates $\left(x^{1}, \ldots, x^{2 n}\right): U \rightarrow \mathbb{R}^{2 n}$ the symplectic form $\omega$ can be written as

$$
\omega=\omega_{j l} d x^{j} \wedge d x^{l} .
$$

Here and later we use the standard convention of summation over repeated indices. Denote by $y^{j}$ the image of $d x^{j}$ in $S\left(T_{x}^{*} M\right)$. Any section $a \in \Gamma\left(W_{\hbar}, U\right)$ is a formal power series:

$$
a=\sum_{l \geq 0} \sum_{\alpha} a_{l, \alpha}(x) y^{\alpha} \hbar^{l}
$$


where $\alpha=\left(\alpha_{1}, \ldots, \alpha_{2 n}\right)$ is a multiindex. In this notation the Moyal-Weyl product of two sections $a, b \in \Gamma\left(W_{\hbar}, U\right)$ is

$$
a \circ b=\left.\exp \left(\frac{\hbar}{2} \omega^{j l}(x) \frac{\partial}{\partial y^{j}} \frac{\partial}{\partial z^{l}}\right) a(y) b(z)\right|_{z=y} .
$$

\subsection{Constructing Fedosov's connections}

The main idea of Fedosov's quantization is to construct a flat connection in the Weyl bundle $W_{\hbar}$ for which the exponential map identifies flat sections with smooth functions on $M$. Then the Moyal-Weyl product (2.9) descends to a star product on $C^{\infty}(M)[[\hbar]]$.

Denote by $\Lambda$ the exterior algebra bundle on $M$ :

$$
\Lambda=\wedge\left(T^{*} M\right)
$$

Moyal-Weyl product on $W_{\hbar}$ together with the exterior product on $\Lambda$ equips $W_{\hbar} \otimes \Lambda$ with a structure of a super algebra. ( $W_{\hbar}$ sits in even degree, $\Lambda^{l}$ as usual in even/odd degree depending on the parity of $l$.) The associative products in $\left(W_{\hbar} \otimes \Lambda\right)_{x}, x \in M$ and $\Gamma\left(W_{\hbar} \otimes \Lambda\right)$ will be again denoted by $\circ$, and the corresponding supper bracket by $[.,$.$] .$

There are two canonical fiberwise endomorphisms of $W_{\hbar} \otimes \Lambda$, defined locally by

$$
\delta(a)=\sum_{j} d x^{j} \wedge \frac{\partial a}{\partial y^{j}}
$$

and

$$
\delta^{-1}(a)=\left\{\begin{array}{cc}
\left.\frac{1}{p+q} \sum_{j} y^{j}\left(\frac{\partial}{\partial x^{j}}\right\lrcorner a\right), & \text { for } a \in\left(W_{\hbar}^{p} \otimes \Lambda^{q}\right)_{x}, p+q>0, \\
0, & \text { for } a \in\left(W_{\hbar}^{0} \otimes \Lambda^{0}\right)_{x} .
\end{array}\right.
$$

They satisfy

$$
\delta^{2}=\left(\delta^{-1}\right)^{2}=0
$$

and a Hodge type relation

$$
a=\left(\delta \delta^{-1}+\delta^{-1} \delta\right) a+a_{00}, \quad \forall a \in\left(W_{\hbar} \otimes \Lambda\right)_{x},
$$

where $a_{00}$ is the component of $a$ in $\left(W_{\hbar}^{0} \otimes \Lambda^{0}\right)_{x}$.

Fix a symplectic torsion free connection on $M$. Let $\partial$ denote its covariant derivative. It extends in a natural way to a derivation of $\left(\Gamma\left(W_{\hbar} \otimes \Lambda\right)\right.$, ०). In local Darboux coordinates on an open $U$

$$
\partial a=d a+\frac{1}{\hbar}[\Gamma, a], \quad a \in \Gamma(W \otimes \Lambda, U),
$$

where

$$
\Gamma=\frac{1}{2} \Gamma_{j k l} y^{j} y^{k} d x^{l}
$$

and $\Gamma_{j k l}$ are the Christoffel symbols of the connection in the considered coordinate system. 
Consider more general derivations of $\left(\Gamma\left(W_{\hbar} \otimes \Lambda\right), \circ\right)$ (or "nonlinear connections" on $W_{\hbar}$ ) of the type

$$
D=\partial-\delta+\frac{1}{\hbar} \operatorname{ad}(\gamma)
$$

with

$$
\gamma \in \oplus_{p \geq 3} \Gamma\left(W_{\hbar}^{p} \otimes \Lambda^{1}\right)
$$

One computes

$$
D^{2} a=\frac{1}{\hbar}[\Omega, a], \forall a \in \Gamma\left(W_{\hbar} \otimes \Lambda\right)
$$

with

$$
\Omega=R+\partial \gamma-\delta \gamma+\frac{1}{\hbar} \gamma^{2}+\omega
$$

called Weyl curvature of $D$ (the term $\omega$ is for convenience only). Here $R$ is essentially the curvature of $\partial$

$$
R=\frac{1}{4} R_{i j k l} y^{i} y^{j} d x^{k} \wedge d x^{l}, \quad R_{i j k l}=\omega_{i m} R_{j k l}^{m} .
$$

The connection $D$ is called a Fedosov's connection when it is flat $\left(D^{2}=0\right)$. That is when

$$
\Omega \in Z\left(\Gamma\left(W_{\hbar} \otimes \Lambda\right)\right)
$$

where $Z\left(\Gamma\left(W_{\hbar} \otimes \Lambda\right)\right)$ denotes the supercenter of $\left(\Gamma\left(W_{\hbar} \otimes \Lambda\right), \circ\right)$. It is clear that

$$
Z\left(\Gamma\left(W_{\hbar} \otimes \Lambda\right)\right)=\Omega(M)[[\hbar]],
$$

where $\Omega(M)=\Gamma(\Lambda, M)$ is the space of differential forms on $M$. So eq. (2.19) is equivalent to $\Omega \in \Omega^{2}(M)[[\hbar]]$. In that case Bianchi identities together with eq. (2.16) imply that $D \Omega=d \Omega=0$, i.e.

$$
\Omega \in Z^{2}(M)[[\hbar]],
$$

where now $Z(M)$ stays for closed forms on $M$.

Theorem 2.3 (Fedosov) For any choice of the Weyl curvature $\Omega \in Z^{2}(M)[[\hbar]]$, $\Omega=\omega+O(\hbar)$, there exists a unique Fedosov's connection on $W$ with

$\gamma \in \oplus_{p \geq 3} \Gamma\left(W_{\hbar}^{p} \otimes \Lambda^{1}\right)[[\hbar]]$ and $\delta \gamma=0$. The components of of the one-form $\gamma$ in the grading (2.6)-(2.7) of $W_{\hbar}$ are consecutively determined from

$$
\gamma=\delta^{-1}\left(-\Omega+\omega+R+\partial \gamma+\frac{1}{\hbar} \gamma^{2}\right)
$$

\subsection{Construction of Fedosov's star products}

For a Fedosov's connection $D$ on $W_{\hbar}$ the space

$$
W_{D}^{\hbar}=\left\{a \in \Gamma\left(W_{\hbar}\right) \mid D a=0\right\}
$$

of flat sections of $\Gamma\left(W_{\hbar}\right)$ is a subalgebra of $\left(\Gamma\left(W_{\hbar}\right), \circ\right)$.

Given a section $a \in \Gamma\left(W_{\hbar}\right)$ denote by $a_{0}$ its component in $S^{0}\left(T^{*} M\right)[[\hbar]]=$ $C^{\infty}(M)[[\hbar]]$, i.e. the constant (in $y$ ) term in (2.8). 
Theorem 2.4 (Fedosov) For any $f \in C^{\infty}(M)[[\hbar]]$ there exists a unique $\sigma(f) \in W_{D}^{\hbar}$ such that $\sigma(f)_{0}=f$. The graded components $\sigma(f)$ with respect to the grading (2.6)(2.7) of $W_{\hbar}$ are determined inductively by

$$
\sigma(f)=f+\delta^{-1}\left(\partial(\sigma(f))+\frac{1}{\hbar}[\gamma, \sigma(f)]\right) .
$$

The above theorem allows to introduce an associative product on $C^{\infty}(M)[[\hbar]]$ by

$$
f * g=(\sigma(f) \circ \sigma(g))_{0}, \quad f, g \in C^{\infty}(M)[[\hbar]] .
$$

One easely sees that it is a deformation of the Poisson structure on $(M, \omega)$.

\subsection{Classification of star products}

The classification of star products on a symplectic manifold was obtained in [6] via cohomological methods developed in [3]. In Fedosov's approach it was done in [9, 15, 19. For comparison between the two approaches see [4]. The classification is summarized in the following theorem.

Theorem 2.5 (i) There exists a bijective correspondence between the set of isomorphism classes of star products on $(M, \omega)$ and the classes of formal deformations of the two-form $\omega$ on $M\left(\equiv\left\{[\Omega] \in H^{2}(M)[[\hbar]] \mid \Omega=\omega+O(\hbar)\right\}\right)$.

(ii) Any star product is equivalent to a Fedosov's one and the class of this product referred to in part $(i)$ is the class of the curvature form $[\Omega]$ of the corresponding Fedosov's connection (see Theorem 2.3).

Using Moser's classification of nearby symplectic structures [13, Theorem 2.5 can be rephrased as follows:

The equivalence classes of star products on $(M, \omega)$ are in one to one correspondence with the equivalence classes of formal $(\mathbb{R}[[\hbar]])$ deformations of the symplectic structure $\omega$ on $M$.

The equivalence relation in the second set is defined by the action of the group of formal symplectomorphisms of $(M, \omega)$ starting with the identity diffeomorphism of $M$.

\subsubsection{Semiclassical Fedosov's exponential map}

Consider the formal bundle $\mathcal{J}^{\infty}(M)$ over $M$ whose fiber over $x \in M$ is the set of $\infty$-jets $\mathcal{J}_{x}^{\infty}(M)$ of real valued functions on $M$ at $x$. For a symplectic manifold $(M, \omega)$ each fiber of $\mathcal{J}^{\infty}(M)$ has a natural Poisson structure and so does each fiber of the Weyl bundle $W$. Emmrich and Weinstein [7 found that a semiclassical analog of Fedosov's construction gives a fiberwise isomorphism

$$
\exp : \mathcal{J}^{\infty}(M) \rightarrow W
$$

Their construction goes as follows. Consider a symplectic torsion free connection $\partial$ on $M$. There exists

$$
\gamma \in \oplus_{p \geq 3} \Gamma\left(W^{p} \otimes \Lambda^{1}\right)
$$


such that

$$
D^{0}=\partial-\delta+\{\gamma, \cdot\}_{\text {fib }}
$$

is a flat connection on $W$, i.e. $\left(D^{0}\right)^{2}=0$. With $\{., .\}_{\text {fib }}$ we denote the fiberwise Poisson bracket on $W$. One such $\gamma$ can be inductively computed from

$$
\gamma=\delta^{-1}\left(R+\partial \gamma+\frac{1}{2}\{\gamma, \gamma\}_{\mathrm{fib}}\right)
$$

(cf. (2.21)). Here $R$ is the curvature of $\partial$, as in eq. (2.18). Denote the jet of flat sections of $W$ with respect to $D^{0}$ at $x \in M$ by

$$
\mathcal{J}_{x}^{\infty}(W)_{D^{0}}=\left\{a \in \mathcal{J}_{x}^{\infty}(W) \mid D^{0} a=0\right\} .
$$

For any $f \in \mathcal{J}_{x}^{\infty}(M)$ there exists a unique $\bar{f} \in \mathcal{J}_{x}^{\infty}(W)_{D^{0}}$ such that

$$
(\bar{f})_{0}=f .
$$

Iteratively it is computed from

$$
\bar{f}=f+\delta^{-1}(\partial(\bar{f})+\{\gamma, \bar{f}\})
$$

cf. (2.23). At the end one can define

$$
\exp _{x}(f)=\bar{f}(x)
$$

From eq. (2.30) it follows that the evaluation in the RHS makes sense.

Proposition 2.6 The map $\exp : \mathcal{J}_{x}^{\infty}(M) \rightarrow(W)_{x}$ defined by 2.31) is a fiberwise Poisson isomorphism.

Proof. From the fact that $D^{0}$ is a differentiation of the Poisson algebra $\Gamma(W)$ and thus differentiation of the algebras $\mathcal{J}_{x}^{\infty}(W)$ for any $x \in M$ it follows that exp is a fiberwise Poisson map. The map exp is a fiberwise isomorphism because

$$
\exp _{x}(f)=f+\frac{\partial f}{\partial x^{j}} y^{j}+O\left(y^{2}\right)
$$

in any coordinate system $\left(x^{1}, \ldots, x^{2 n}\right)$ in a neighborhood of $x \in M$.

\subsubsection{A proof of the classification Theorem}

Here we explain some parts of the proof of Theorem 2.5. In particular following P. Xu [19] we show how one obtains from a given star product $*$ on $(M, \omega)$ a Fedosov's star product equivalent to it. The product $*$ is a local one and thus it induces a star product on $\mathcal{J}_{x}^{\infty}(M), x \in M$. Using the Emmrich-Weinstein exponential map exp we get a fiberwise star product $\bullet$ on $W_{\hbar}$ :

$$
a \bullet b:=\exp _{x}\left(\exp _{x}^{-1}(a) * \exp _{x}^{-1}(b)\right), \text { for } a, b \in(W)_{x},(x \in M) .
$$


This product is a deformation quantization of the fiberwise Poisson algebra structure $\{., .\}_{\text {fib }}$ on $W$ due to Proposition 2.6. It is fiberwise equivalent to the MoyalWeyl product $\circ$ (2.9), because $T M$ is a regular Poisson manifold with contractable symplectic leaves. Therefore there exists a differential operator of the form

$$
P=1+\sum_{l=1}^{\infty} \hbar^{l} P_{l},
$$

where $P_{l}$ are differential operators on $T M$ that involve derivatives along the tangent spaces only, such that

$$
P(a \bullet b)=P(a) \circ P(b), \quad \forall a, b \in \Gamma\left(W_{\hbar}\right) .
$$

Let

$$
j: C^{\infty}(M) \rightarrow \Gamma\left(\mathcal{J}^{\infty}(M)\right)
$$

be the natural evaluation map, sending a smooth function to the $\infty$-jet that it determines at each point of $M$. Then the composition

$$
\rho=P \cdot \exp \cdot j:\left(C^{\infty}(M)[[\hbar]], *\right) \rightarrow\left(\Gamma\left(W_{\hbar}\right), \circ\right)
$$

is an injective homomorphism having the following properties

(i) $(\rho(f))_{0}=f$,

(ii) $\rho(f) \equiv f+\delta^{-1} d f\left(\bmod \Gamma\left(W_{\hbar}^{2}\right)\right)$.

Any Fedosov's connection $D$ gives rise to a map $\sigma_{D}$ defined in Theorem 2.4, which satisfies (i)-(ii). P. Xu proved a converse statement.

Theorem 2.7 Let $*$ be a star product on $M$ and $\rho:\left(C^{\infty}(M)[[\hbar]], *\right) \rightarrow\left(\Gamma\left(W_{\hbar}\right), \circ\right)$ be a homomorphism that satisfies (i)-(ii). Then there exists a Fedosov's connection D such that

$$
\rho=\sigma_{D}
$$

This Theorem applied to the composition (2.34) gives a Fedosov's connection $D$ on $W_{\hbar}$ and a Fedosov's star product $*_{F}$ on $M$ equivalent to the initial one $*$. The class of the curvature form of $D$ is called characteristic class of the star product $*$ and is the two-form that appears in Theorem 2.5.

Finally two Fedosov's products are equivalent if and only if their characteristic classes are equal. We will not need a proof of this fact here. The reader can find it in [9, 15].

\section{Lagrangian fiber bundles and some connections asso- ciated with them}

Let $(M, \omega)$ be a symplectic manifold and $\pi: M \rightarrow B$ be a Lagrangian fiber bundle, i.e $\pi$ is a fiber bundle whose fibers are Lagrangian submanifold of $(M, \omega)$. We assume also that the fibers of $\pi$ are connected and that for each $f \in C^{\infty}(B)$ the Hamiltonian vector field $H\left(\pi^{*} f\right)$ is complete. Equivalently the fibers of $\pi$ should be diffeomorphic to $\mathbb{R}^{n} / \mathbb{Z}^{k}$ for some $k \leq n$. We do not require compactness of the fibers thus $k$ can be $<n$. 


\subsection{Action-angle coordinate charts}

In this subsection we review the construction of action-angle coordinate charts on $M$ (see [1, 11, 5]).

For any $b \in B$ denote

$$
M^{b}=\{x \in M \mid \pi(x)=b\} .
$$

$T_{b}^{*} B$ acts on $M^{b}$ in the following way. Let $f$ be a smooth function defined in a neighborhood of $b$. The action of $(d f)_{b}$ on $x \in M^{b}$ is defined by

$$
\tau\left((d f)_{b}\right) x=g_{1}^{H\left(\pi^{*} f\right)}(x),
$$

where on the RHS $g$ denotes the flow on $M$ corresponding to a Hamiltonian vector field (upper index) at a given time (lower index). This flow preserves $M^{b}$ and thus it depends on $(d f)_{b}$ only, so the action is correctly defined. $T_{b}^{*} B$ is a commutative group, thus the stabilizers of all points of $M^{b}$ coincide. Denote the corresponding subgroup of $T_{b}^{*} B$ by $P_{b}$. It is obvious that $P_{b} \cong \mathbb{Z}^{k}$ for some $k \leq n$. Varing $b$ we get a submanifold $P$ of $T^{*} B$. It is also clear that it is a Lagrangian submanifold of $T^{*} B$. The action of $T_{b}^{*} B$ on $M^{b}$ defines a faithful action of the fibers $T_{b}^{*} B / P_{b}$ of $T^{*} B / P$ on the fibers $M^{b}$ of the bundle $\pi$.

Let $U \subset B$ be an open set for which there exists a section

$$
s: U \rightarrow M
$$

of $\pi(\pi \circ s=\mathrm{id})$, such that

1. $s(U)$ is a Lagrangian submanifold of $M$

2. $s$ is a diffeomorphism onto its image.

The two conditions roughly mean that we consider a Lagrangian submanifold $s(U)$ transversal to the fibration. Any such section determines a symplectic diffeomorphism

$$
\left.\pi^{-1}(U) \cong\left(T^{*} B / P\right)\right|_{U},
$$

where $T^{*} B$ is equipped with the standard symplectic structure as a cotangent bundle. To prove this simply act with $T_{b}^{*} B / P_{b}$ on $s(b)$ for $b \in U$.

In the case when $U$ is diffeomorphic to a domain in $\mathbb{R}^{n}$ trivializing $\left.\left(T^{*} B / P\right)\right|_{U}$ gives a chart on $\pi^{-1}(U)$ of the type

$$
\left(I^{1}, I^{2}, \ldots, I^{n}, \varphi^{1}, \varphi^{2}, \ldots, \varphi^{n}\right): \pi^{-1}(U) \rightarrow O \times\left(\mathbb{R}^{n} / \mathbb{Z}^{k}\right) .
$$

Here $O$ is an open domain of $\mathbb{R}^{n}$, which from now on will be denoted by the same letter $U$. The lattice $\mathbb{Z}^{k}$ is assumed to be embedded in the standard way in $\mathbb{R}^{k} \subset \mathbb{R}^{n}$ spanned by the first $k$ coordinate vectors. The coordinates $(I, \varphi)$ are called actionangle coordinates, although in the case $k<n$ only $\varphi^{1}, \ldots, \varphi^{k}$ are angles. Each $b \in B$ has a neighborhood of the above type and thus $M$ can be covered by action-angle charts. 
Let

$$
\left(\bar{I}^{1}, \bar{I}^{2}, \ldots, \bar{I}^{n}, \bar{\varphi}^{1}, \bar{\varphi}^{2}, \ldots, \bar{\varphi}^{n}\right): \pi^{-1}(\bar{U}) \rightarrow \bar{U} \times\left(\mathbb{R}^{n} / \mathbb{Z}^{k}\right) .
$$

be another action-angle chart on $\pi^{-1}(\bar{U})$ for some open domain $\bar{U}$ with $U \cap \bar{U} \neq \emptyset$. The change of coordinates on the intersection of the two action-angle charts is of a particularly simple form:

$$
\begin{aligned}
\bar{I}^{\alpha} & =f^{\alpha}(I), \quad \alpha=1, \ldots, n, \\
\bar{\varphi}^{j} & =A_{l}^{j}(I) \varphi^{l}+B^{j}(I), \quad i, \alpha=1, \ldots, n,
\end{aligned}
$$

where $A_{l}^{j}(I)$ is the inverse of the matrix $\frac{\partial \bar{I}^{l}}{\partial I^{l}}$ and $f^{\alpha}, B^{1}, \ldots, B^{n}$ are some $C^{\infty}$ functions.

The RHS of (3.8) is linear in $\varphi^{l}$ because of the way we defined the identification (3.4). $B^{j}(I), j=1, \ldots, n$ are the coordinates of the Lagrangian section $\left.s\right|_{U \cap \bar{U}}$ of $\pi$ (see $(3.3))$ in the action-angle chart $(\bar{I}, \bar{\varphi})$ on $\pi^{-1}(U \cap \bar{U})$ :

$$
s(\bar{I})=\left(\bar{I}, B^{j}(I(\bar{I}))\right) .
$$

This imposes some standard differential conditions on the functions $B^{j}(I)$.

Note also that (3.8) should define a map from $\mathbb{R}^{n} / \mathbb{Z}^{k}$ to $\mathbb{R}^{n} / \mathbb{Z}^{k}$. This means that $A_{l}^{j}(I)+B^{j}(I)$ should be integers for $j=1, \ldots n, l=1, \ldots k$ and thus constants as functions of the action variables $I$.

\subsection{Special symplectic connections}

In the second part of of this section we consider certain type of symplectic connections associated to a Lagrangian fiber bundle $\pi: M \rightarrow B$. They will be used in Sections 4 and 5 as initial connections $\partial$ for special Fedosov's connections.

First we give a computational proof of our main theorem and then explain some parts of it in geometric terms.

Theorem 3.1 There exists a torsion free symplectic connection $\Gamma$ on $M$ such that in each action-angle chart $(I, \varphi)$ as in (3.5) the Christoffel symbols of $\Gamma$ satisfy the following properties:

$$
\begin{aligned}
& \Gamma_{\varphi^{j} \varphi^{l}}^{I^{\alpha}}=0, \text { i.e. } \Gamma_{\varphi^{j} \varphi^{l} \varphi^{m}}=0, \\
& \Gamma_{\varphi^{j} \varphi^{l}}^{\varphi^{m}}=\Gamma_{I^{\alpha} \varphi^{j}}^{I^{\beta}}=0 \text {, i.e. } \Gamma_{I^{\alpha} \varphi^{j} \varphi^{l}}=0, \\
& \left.\Gamma_{I^{\alpha} \varphi^{j}}^{\varphi^{l}}, \Gamma_{I^{\alpha} I^{\beta}}^{I^{\gamma}} \text { (and thus also } \Gamma_{I^{\alpha} I^{\beta} \varphi^{j}}\right) \text { do not depend on } \varphi^{m}, \\
& \left.\Gamma_{I^{\alpha} I^{\beta}}^{\varphi^{j}}, \quad \text { (and thus also } \Gamma_{I^{\alpha} I^{\beta} I^{\gamma}}\right) \text { are liner in } \varphi^{m} .
\end{aligned}
$$

$\forall j, k, l, m, \alpha, \beta$, and $\gamma=1, \ldots, n$.

Here and later Greek letters will be used as indices for action coordinates and Latin letters as indices for angle coordinates.

Proof. We construct a connection with the required properties by chart extension. Suppose that we have defined the connection on a union $\pi^{-1}(\mathcal{U})$ of action-angle 
charts $\pi^{-1}(U)(3.5)$ and we would like to extend it to $\pi^{-1}(\bar{U})$, with action-angle coordinates as in (3.6). The Christoffel symbols for $\Gamma$ on it will be defined consecutively in the order listed in the statement of the theorem.

(1) Because of the transformation rules (3.7)-(3.8) the symbols $\Gamma_{\bar{\varphi}^{j} \bar{\varphi}^{l}}^{\bar{I}^{\alpha}}$ of $\Gamma$ should satisfy

$$
\Gamma_{\bar{\varphi}^{j} \bar{\varphi}^{l}}^{\bar{I}^{\alpha}}=\sum_{j^{\prime}, l^{\prime}, \alpha^{\prime}} \frac{\partial \bar{I}^{\alpha}}{\partial I^{\alpha^{\prime}}} \frac{\partial \varphi^{j^{\prime}}}{\partial \bar{\varphi}^{j}} \frac{\partial \varphi^{l^{\prime}}}{\partial \bar{\varphi}^{l}} \Gamma_{\varphi^{j^{\prime}} \varphi^{l^{\prime}}}^{I^{\alpha^{\prime}}}
$$

on $\pi^{-1}(U \cap \bar{U})$, for $U \subset \mathcal{U}$. But $\Gamma$ satisfies (3.9) on $\pi^{-1}(U)$, so we are free to put $\Gamma_{\bar{\varphi}^{j} \bar{\varphi}^{\alpha}}^{\bar{I}^{\alpha}}=0$ on $\pi^{-1}(\bar{U})$.

(2) Similarly to part (1) we can define

$$
\Gamma_{\bar{\varphi}^{j} \bar{\varphi}^{l}}^{\bar{\varphi}^{m}}=\Gamma_{\bar{I}^{\alpha} \bar{\varphi}^{j}}^{\bar{I}^{\beta}}=0
$$

on $\pi^{-1}(\bar{U})$ and this will be consistent with the definition of $\Gamma$ on $\pi^{-1}(\mathcal{U})$.

(3) The transformation property that $\Gamma_{\bar{I}^{\alpha} \bar{\varphi}^{j}}^{\bar{\varphi}^{l}}$ should satisfy is

$$
\begin{aligned}
\Gamma_{\bar{I}^{\alpha} \bar{\varphi}^{j}}^{\bar{\varphi}^{l}} & =\sum_{\alpha^{\prime} j^{\prime} l^{\prime}} \frac{\partial I^{\alpha^{\prime}}}{\partial \bar{I}^{\alpha}} \frac{\partial \varphi^{j^{\prime}}}{\partial \bar{\varphi}^{j}} \frac{\partial \bar{\varphi}^{l}}{\partial \varphi^{l^{\prime}}} \Gamma_{I^{\alpha^{\prime}} \varphi^{j^{\prime}}}^{l^{l^{\prime}}} \\
& +\sum_{\alpha^{\prime} j^{\prime} \beta^{\prime}} \frac{\partial I^{\alpha^{\prime}}}{\partial \bar{I}^{\alpha}} \frac{\partial \varphi^{j^{\prime}}}{\partial \bar{\varphi}^{j}} \frac{\partial \bar{\varphi}^{l}}{\partial I^{\beta^{\prime}}} \Gamma_{I^{\alpha^{\prime}} \varphi^{j^{\prime}}}^{\beta^{\prime}} \\
& +\sum_{\alpha^{\prime} j^{\prime} \beta^{\prime}} \frac{\partial \varphi^{m^{\prime}}}{\partial \bar{I}^{\alpha}} \frac{\partial \varphi^{j^{\prime}}}{\partial \bar{\varphi}^{j}} \frac{\partial \bar{\varphi}^{l}}{\partial \varphi^{l^{\prime}}} \Gamma_{\varphi^{m^{\prime}} \varphi^{j^{\prime}}}^{l^{\prime}} \\
& +\sum_{\beta^{\prime} j^{\prime} m^{\prime}} \frac{\partial \varphi^{m^{\prime}}}{\partial \bar{I}^{\alpha}} \frac{\partial \varphi^{j^{\prime}}}{\partial \bar{\varphi}^{j}} \frac{\partial \bar{\varphi}^{l}}{\partial I^{\beta^{\prime}}} \Gamma_{\varphi^{m^{\prime}} \varphi^{j^{\prime}}}^{\beta^{\beta^{\prime}}} \\
& +\sum_{l^{\prime}} \frac{\partial^{2} \varphi^{l^{\prime}}}{\partial \bar{I}^{\alpha} \partial \bar{\varphi}^{j}} \frac{\partial \bar{\varphi}^{l}}{\partial \varphi^{l^{\prime}}}
\end{aligned}
$$

on $\pi^{-1}(U \cap \bar{U})$, for $U \subset \mathcal{U}$. Eqs. (3.9)-(3.11) imply that on $\pi^{-1}(U \cap \bar{U}) \Gamma_{\varphi^{m^{\prime}} \varphi^{j^{\prime}}}^{{\beta^{\prime}}^{\prime}}=$ $\Gamma_{I^{\alpha^{\prime}} \varphi^{j^{\prime}}}^{I^{\beta^{\prime}}}=\Gamma_{\varphi^{m^{\prime}} \varphi^{j^{\prime}}}^{\varphi^{l^{\prime}}}=0$ and $\Gamma_{I^{\alpha^{\prime}} \varphi^{j^{\prime}}}^{\varphi^{l^{\prime}}}$ do not depend on $\varphi$. From this it is clear that the RHS of (3.13) is a function on $\pi^{-1}(U \cap \bar{U})$, which does not depend on $\varphi$ (see also (3.7)-(3.8)). It can be used to define $\Gamma_{\bar{I}^{\alpha} \bar{\varphi}^{j}}^{\bar{\varphi}^{l}}$ on $\pi^{-1}(\mathcal{U} \cap \bar{U})$, and at the end we can define them on $\pi^{-1}(\bar{U})$ by any continuation from the closure of $\pi^{-1}(U \cap \bar{U})$.

One deals with the symbols $\Gamma_{\bar{I}^{\alpha} \bar{I}^{\beta}}^{\bar{I}^{\gamma}}$ in the same way.

(4) To define $\Gamma_{\bar{I}^{\alpha} \bar{I}^{\beta}}^{\bar{\varphi}^{j}}$ on $\pi^{-1}(\bar{U})$ so that (3.12) holds, one just repeates the construction from (3).

From this construction we can get a torsion free symplectic connection satisfying (3.9)-(3.12). This can be done in two ways. First, it is easy to keep on each step the symbols $\Gamma_{x y z}$ completely symmetric. The second option is to construct a connection $\Gamma$ with the stated properties for $\Gamma_{x y}^{z}$ and then to symmetrize $\Gamma_{x y z}$. The result is a connection with all the properties from Theorem 3.1. 
Remark 3.2 One can interprete properties (3.9)-(3.11) in a more geometric language.

For each $x \in M, b \in B, T_{x} M^{b} \subset T_{x} M$ can be canonically identified with $T_{b}^{*} B$, by linearization of the action of $T_{b}^{*} B / P_{b}$ on $M^{b}$. Denote this identification by

$$
i_{x}: T_{x} M^{b} \rightarrow T_{b}^{*} B
$$

Let $\Gamma_{B}$ and $\Gamma_{M}$ be two connections on $B$ and $M$ respectively with the following property.

If $y \in M_{b^{\prime}}, b^{\prime} \in B$ and $\gamma$ is a curve connecting $x$ and $y$ then

$$
\mathcal{T}_{\gamma}^{M}(e)=i_{y}^{-1}\left(\mathcal{T}_{\pi(\gamma)}^{B}\left(i_{x}(e)\right)\right)
$$

where $\mathcal{T}^{M}$ and $\mathcal{T}^{B}$ denote the parallel translations of tangent vectors to $M$ and cotangent vectors to the base $B$ along the specified curves (corresponding to $\Gamma_{M}$ and $\left.\Gamma_{B}\right)$.

Such a connection $\Gamma_{M}$ satisfies properties 3.9$)-(3.10)$ because for $\gamma \subset M^{b}$

$$
\mathcal{T}_{\gamma}^{B} \equiv i_{y}^{-1} i^{x}: T_{x} M^{b} \cong T_{y} M^{b},
$$

i.e. $M^{b}$ is a flat submanifold. Property (3.11) also holds for $\Gamma_{M}$, because the parallel translation $\mathcal{T}^{M}$ of vectors in $T_{x} M^{b}$ comes from the translation $\mathcal{T}^{B}$ on the base (see (3.14)).

Eq. (3.14) can be actually used to define $\Gamma_{M}$ on $T_{x} M^{b}$, and it will satisfy (3.9)(3.11). This argument can substitute parts (1)-(3) of the above proof.

Remark 3.3 $P$ is a covering of $B$ and we can define parallel translation of the vectors of the lattice $P_{b} \subset T_{b}^{*} B$ along any curve in $B$. Because of the nature of $P\left(P_{b}\right.$ is the kernel of the action $\tau$ (3.2) of $T_{b}^{*} B$ on $\left.M_{b}\right)$ this can be extended by linearity to parallel translation of vectors in $\operatorname{span}_{b} \subset T_{b}^{*} B$. In the special case when $\pi$ has compact fibers $(k=n)$ it gives a torsion free flat connection on $B$. Using it as described in the previous remark we get a connection on $M$ for which all Christoffel symbols from (3.9)-(3.11) vanish.

\section{Existence of star products preserving a Lagrangian fiber bundle}

The goal of this section is to prove the existence of nontrivial deformations of $C^{\infty}(M)$, that keep $\pi^{*}\left(C^{\infty}(B)\right)$ undeformed. In the next section we show that up to equivalence of star products these are all deformations of $C^{\infty}(M)$ in which $\pi^{*}\left(C^{\infty}(B)\right)$ generates a commutative subalgebra.

Theorem 4.1 Let $\Omega \in Z^{2}(M)[[\hbar]]$ be a formal deformation of the symplectic form $\omega$ :

$$
\Omega=\omega+O(\hbar)
$$


such that $\pi: M \rightarrow B$ is a Lagrangian fibration with respect to $\Omega$, that is

$$
\left.\Omega\right|_{\pi^{-1}(b)}=0, \forall b \in B
$$

Then there exists a Fedosov's star product with characteristic class $[\Omega]$ that keeps $\pi^{*}\left(C^{\infty}(B)\right)$ undeformed.

Denote by $\bar{W}_{B}$ the Weyl bundle on $B$ and by $\Gamma\left(W_{B}\right)$ the pull back to $M$ of the space of its global sections:

$$
\Gamma\left(W_{B}\right)=\pi^{*}\left(\Gamma\left(\bar{W}_{B}\right)\right) \subset \Gamma\left(W_{M}\right)
$$

Consider also the conormal bundle to the fibration $\pi$

$$
\left(N_{\pi}^{*}\right)_{x}=\left\{v \in T_{x}^{*} M \mid<v, e>=0, \forall e \in T_{x} M^{b}\right\} \quad \text { for } x \in M^{b}, b \in B .
$$

We will call

$$
W_{\pi}=S\left(N_{\pi}^{*}\right) \subset W_{M}
$$

Weyl bundle of the fibration $\pi$. Also denote $W_{B, \hbar}=W_{B}[[\hbar]], W_{\pi, \hbar}=W_{\pi}[[\hbar]]$, and $W_{M, \hbar}=W_{M}[[\hbar]]$. The following inclusions are obvious

$$
\Gamma\left(W_{B}\right) \subset \Gamma\left(W_{\pi}\right) \subset \Gamma\left(W_{M}\right)
$$

and clearly $\Gamma\left(W_{\pi}\right)$ is an undeformed subalgebra of $\Gamma\left(W_{M, \hbar}\right)$ with respect to the fiberwise Moyal-Weyl product (2.9).

Locally, in action-angle coordinates $(I, \varphi)$ on some $\pi^{-1}(U)$ as in eq. (3.5) denote by $J^{\beta}$ and $\psi^{j}$ the images of $d I^{\beta}$ and $\varphi^{j}$ in $W_{M}=S\left(T^{*} M\right)$ for $\beta, j=1, \ldots, n$. In this notation $\Gamma\left(W_{\pi, \hbar}, \pi^{-1}(U)\right)$ consists of sections (2.8) that are power series in $J^{\beta}$ only and $\pi^{*}\left(\Gamma\left(\bar{W}_{B, \hbar}, U\right)\right)$ of the subspace of sections for which in addition $a_{k, \alpha}$ depend on the action coordinates $I^{\beta}$ only.

In Subsect. 4.1 we show that for any closed two-form $\Omega$ as in the statement of Theorem 4.1 there exists a closed form $\Omega^{\prime}$ of a special type cohomologous to it. In the second subsection we deduce Theorem 4.1 from a stronger result stating that Fedosov's construction applied to $\Omega^{\prime}$ exponentiates $\pi^{*}\left(C^{\infty}(B)\right)$ to a subalgebra of $\Gamma\left(W_{B, \hbar}\right)$. The latter, as we remarked above, is an undeformed subalgebra of $\left(\Gamma\left(W_{M, \hbar}\right), \circ\right)$.

\subsection{Construction of special closed two-forms}

Denote by $\Gamma\left(W_{M, \hbar} \otimes \Lambda\right)^{\text {pol }}$ the subalgebra of $\Gamma\left(W_{M, \hbar} \otimes \Lambda\right)$ consisting of those sections which in any action-angle chart (3.5) are formal power series

$$
\sum_{l=0}^{\infty} \sum_{\alpha, \gamma} \hbar^{l} f_{a, \gamma, l}(I, \varphi) y^{\alpha} d x^{\gamma}
$$

with $f_{a, \gamma, l}(I, \varphi)$ being polynomials in the variables $\varphi^{k+1}, \ldots, \varphi^{n}$, where $x=(I, \varphi)$, $y=(J, \psi)$, and $d x^{\gamma}=d x^{\gamma_{1}} \wedge \ldots \wedge d x^{\gamma_{s}}$ (see also the remarks to eq. (3.5)). The above 
requirement does not depend on the choice of a particular action-angle coordinate system $(I, \varphi)$ on an open $\pi^{-1}(U)$ because of the transformation rules (3.7)-(3.8). The same formulas make possible to define a filtration on $\Gamma\left(W_{M, \hbar} \otimes \Lambda\right)^{\text {pol }}$ by letting (locally)

$$
\operatorname{deg} \varphi^{j}=\operatorname{deg} d \varphi^{j}=\operatorname{deg} \psi^{j}=1, \quad j=1, \ldots, n .
$$

Denote the part of $\Gamma\left(W_{M, \hbar} \otimes \Lambda\right)^{\text {pol }}$ of deg $\leq l$ by

$$
\Gamma\left(W_{M, \hbar} \otimes \Lambda\right)^{(l)}
$$

(not to be mistaken with the grading $(2.6)$ ). In the same way we define the subspaces $\Gamma\left(W_{M, \hbar}\right)^{\mathrm{pol}}$ and $\Gamma(\Lambda)^{\mathrm{pol}}$ of $\Gamma\left(W_{M, \hbar} \otimes \Lambda\right)^{\mathrm{pol}}$ and restrict the filtration (4.8) to them.

We are ready to state the main result of this subsection.

Proposition 4.2 Let $\Omega \in Z^{2}(M)$ and

$$
\left.\Omega\right|_{\pi^{-1}(b)}=0
$$

for all $b \in B$. Then there exists $\Omega^{\prime} \in Z^{2}(M)$ cohomologous to $\Omega$ such that

$$
\Omega^{\prime} \in \Gamma\left(\Lambda^{2}\right)^{(1)} \text {. }
$$

(Then also automatically $\left.\Omega^{\prime}\right|_{\pi^{-1}(b)}=0, \forall b \in B$.)

Both the above result and the Lemma that we need for its proof are well known but we will sketch two elementary proofs of them for completeness.

Lemma 4.3 (i) Let $\alpha \in Z^{2}\left(U \times \mathbb{R}^{n}\right)$ for an open domain $U$ in $\mathbb{R}^{n}$. Assume that

$$
\left.\alpha\right|_{\pi_{1}^{-1}(u)}=0, \forall u \in U
$$

where $\pi_{1}: U \times \mathbb{R}^{n} \rightarrow U$ is the projection on the first factor. Then there exists a one-form $\beta$ such that

$$
\left.\beta\right|_{\pi_{1}^{-1}(u)}=0, \forall u \in U \quad \text { and } \quad d \beta=\alpha .
$$

(ii) If $\beta \in Z^{1}\left(U \times \mathbb{R}^{n}\right)$ and

$$
\left.\beta\right|_{\pi_{1}^{-1}(u)}=0, \forall u \in U
$$

for an open subset $U \subset \mathbb{R}^{n}$ then

$$
\beta=\pi^{*}\left(\beta_{1}\right),
$$

for some $\beta_{1} \in Z^{1}(U)$. 
Proof. Part (i) Denote by $\left(I^{1}, \ldots, I^{n}\right)$ and $\left(\varphi^{1}, \ldots, \varphi^{n}\right)$ the coordinates on $U$ and $\mathbb{R}^{n}$ respectively. If

$$
\alpha=f_{j l}(I, \varphi) d I^{j} \wedge d I^{l}+g_{j l}(I, \varphi) d \varphi^{j} \wedge d I^{l}
$$

then $\alpha^{0}=f_{j l}(I, 0) d I^{j} \wedge d I^{l}$ is closed in $U$ and there exists a one-form $\beta^{0}=a_{l}(I) d I^{l}$ such that $d \beta^{0}=\alpha^{0}$. Using the assumption that $\alpha$ is closed it is easy to prove that

$$
\beta=\beta^{0}+\left(\int_{0}^{\varphi} g_{j l}(I, \phi) d \phi^{j}\right) d I^{l}
$$

satisfies (4.11).

Part (ii) If $\beta=b_{l}(I, \varphi) d I^{l}$ then $d \beta=0$ implies $d_{\varphi} b_{l}(I, \varphi)=0, \forall I \in U$. Thus $b_{l}(I, \varphi)$ depends on $I$ only and we can put $\beta_{1}=b_{l}(I) d I^{l}$.

Proof of Proposition 4.9. We construct a two-form $\Omega^{\prime}$, satisfying (4.9) and a one-form $\gamma$ such that $\Omega-\Omega^{\prime}=d \gamma$ by chart extension.

Let $(I, \varphi)$ be an action-angle chart on some $\pi^{-1}(U) \cong U \times\left(\mathbb{R}^{n} / \mathbb{Z}^{k}\right)$. Denote

$$
\Omega_{U}=\left.\Omega\right|_{\pi^{-1}(U)}
$$

and let its pull back to $U \times \mathbb{R}^{n}$ be

$$
\widetilde{\Omega}_{U}=f_{j l}(I, \varphi) d I^{j} \wedge d I^{l}+g_{j l}(I, \varphi) d \varphi^{j} \wedge d I^{l}
$$

(here $f_{j l}$ and $g_{j l}$ are $\mathbb{Z}^{k}$ periodic in $\left.\varphi^{1}, \ldots, \varphi^{k}\right)$. Lemma 4.3 applied to $\widetilde{\Omega}_{U}$ gives a one-form $\tilde{\beta}_{U}=a_{l}(I, \varphi) d I^{l}$ such that $d \tilde{\beta}_{U}=\widetilde{\Omega}_{U}$. For any $l$ and $I=c=\left(c^{1}, \ldots, c^{n}\right)=$ const

$$
g_{j l}(c, \varphi) d \varphi^{j} \in Z^{2}\left(\mathbb{R}^{n} / \mathbb{Z}^{k}\right)
$$

because $\Omega$ is closed. $H^{2}\left(\mathbb{R}^{n} / \mathbb{Z}^{k}\right) \cong \mathbb{R}^{k}$ and there exists a one-form with coefficients that do not depend on the angle variables $\varphi$

$$
\sum_{j} a_{j l}^{\prime}(c) d \varphi^{j} \sim g_{j l}(c, \varphi) d \varphi^{j}
$$

where the first sum is over $1 \leq j \leq k$, i.e. just coordinates $\varphi^{1}, \ldots, \varphi^{k}$ related to the lattice $\mathbb{Z}^{k}$ appear. Then

$$
\gamma_{U}:=\left(a_{l}(I, \varphi)-\sum_{j} a_{j l}^{\prime}(I) \varphi^{j}\right) d I^{l}
$$

descends to a one-form on $U \times \mathbb{R}^{n} / \mathbb{Z}^{k}$, and so does

$$
\Omega_{U}^{\prime}:=\Omega_{U}-d \gamma_{U}=d\left(\left(\sum_{j} a_{j l}^{\prime}(I) \varphi^{j}\right) d I^{l}\right)
$$

(to a two-form on $U \times \mathbb{R}^{n} / \mathbb{Z}^{k}$ ). Clearly

$$
\Omega_{U}^{\prime} \in \Gamma\left(\Lambda^{2}, \pi^{-1}(U)\right)^{(1)} .
$$


Suppose now that we have already constructed $\Omega^{\prime}$ and $\gamma$ on a union of actionangle charts and we want to extend them to an open $\pi^{-1}(U)$ on $M$ as in (3.5). The intersection of $\pi^{-1}(U)$ with this union is $\pi^{-1}\left(U^{0}\right)$, for some open $U^{0} \subset U$. Denote the restriction of $\gamma$ to $\pi^{-1}\left(U^{0}\right) \cong U^{0} \times \mathbb{R}^{n} / \mathbb{Z}^{k}$ by $\gamma_{U^{0}}$. The second part of Lemma 4.3 together with the transformation rules (3.7)-(3.8) imply that

$$
\gamma_{U^{0}}=\left(a_{l}(I, \varphi)-a_{j l}^{\prime}(I) \varphi^{j}-d_{l}(I)\right) d I^{l}
$$

for some functions $d_{l}(I)$ on $U^{0}$ (the sum over $j$ is from 1 to $k$ again). Finally we can extend $d_{l}(I)$ to $C^{\infty}$-functions on $U$ from the closure of $U^{0}$ and define an extension $\gamma_{U}$ of $\gamma_{U^{0}}$ to $\pi^{-1}(U)$ by the same formula (4.16). $\Omega_{U^{0}}^{\prime}$ also can be extended to $\pi^{-1}(U)$ by $\Omega_{U}^{\prime}=\Omega_{U}-d \gamma_{U}$. Locally on $\pi^{-1}(U)$

$$
\left.\Omega^{\prime}\right|_{U}=d\left(a_{j l}(I) \varphi^{j}+d_{l}(I)\right) \wedge d I^{l} \in \Gamma\left(\Lambda^{2}, \pi^{-1}(U)\right)^{(1)} .
$$

\subsection{Construction of star products preserving a Lagrangian fiber bundle}

The next theorem is the main result in this subsection. It makes Theorem 4.1 more precise, the latter is a consequence of it due to Proposition 4.2 .

Theorem 4.4 Let $\Omega^{\prime} \in Z^{2}(M)[[\hbar]]$ be a formal deformation of $\omega,\left(\Omega^{\prime}=\omega+O(\hbar)\right)$ with the property from Proposition 4.9

$$
\Omega^{\prime} \in \Gamma\left(\Lambda^{2} M[[\hbar]]\right)^{(1)} .
$$

Then any Fedosov's connection D with curvature $\Omega^{\prime}$ based on a symplectic connection $\partial$ with the properties of Theorem 3.1 exponentiates $\pi^{*}\left(C^{\infty}(B)\right)[[\hbar]]$ to a subalgebra of $\Gamma\left(W_{B, \hbar}\right)$ :

$$
\sigma_{D}\left(\pi^{*}\left(C^{\infty}(B)\right)[[\hbar]]\right) \subset \Gamma\left(W_{B, \hbar}\right) .
$$

We will need a simple Lemma.

Lemma 4.5 Assume that $a \in \Gamma\left(W_{M, \hbar}\right)^{(q)}$ and $b \in \Gamma\left(W_{M, \hbar}\right)^{(r)}$, then

(i) $[a, b] \in \Gamma\left(W_{M, \hbar}\right)^{(q+r-1)}$,

(ii) $\partial a \in \Gamma\left(W_{M, \hbar}\right)^{(q)}$, for any connection $\partial$ with the properties of Theorem 3.1,

(iii) $\delta a$ and $\delta^{-1} a \in \Gamma\left(W_{M, \hbar}\right)^{(q)}$.

Proof. Parts (i) and (iii) are trivial. Part (ii) follows from

$$
\begin{aligned}
\partial I^{\alpha} & =\Gamma_{I^{\beta} I^{\gamma}}^{I^{\alpha}} J^{\beta} d I^{\gamma} \in \Gamma\left(W_{M, \hbar}\right)^{(0)}, \\
\partial \varphi^{l} & =\Gamma_{I^{\beta} \varphi^{j}}^{\varphi^{l}} J^{\beta} d \varphi^{l}+\Gamma_{\varphi^{j} I^{\beta}}^{\varphi^{l}} \psi^{j} d I^{\beta}+\Gamma_{I^{\alpha} I^{\beta}}^{\varphi^{l}} J^{\alpha} d I^{\beta} \in \Gamma\left(W_{M, \hbar}\right)^{(1)}
\end{aligned}
$$

for any connection $\partial$ satisfying (3.9)-(3.12).

Proof of Theorem 4.4. Eq. (2.13) implies that the curvature form $R$ of $\partial$ (see (2.18)) in local Darboux coordinates is

$$
R=\partial \Gamma+\frac{1}{\hbar}(\Gamma \circ \Gamma)
$$


where $\Gamma=(1 / 2) \Gamma_{i j l} y^{i} y^{k} d x^{l}$ is the connection form of $\partial$ in the considered coordinate system. Clearly from (3.9)-(3.12) we have

$$
\Gamma \in \Gamma\left(\left(W \otimes \Lambda^{1}\right)^{\mathrm{pol}}, \pi^{-1}(U)\right)^{(1)}
$$

and from Lemma 4.5 we get that

$$
R \in \Gamma\left(W_{M} \otimes \Lambda^{2}\right)^{(1)}
$$

(see Lemma 4.5). Using the same Lemma again from eq. (2.21) we obtain

$$
\gamma \in \Gamma\left(W_{M, \hbar} \otimes \Lambda^{1}\right)^{(1)} .
$$

Finally Lemma 4.5 applied to (2.23) gives

$$
\sigma(f) \in \Gamma\left(W_{M, \hbar}\right)^{(0)}=\Gamma\left(W_{B, \hbar}\right), \forall f \in \pi^{*}\left(C^{\infty}(B)\right) .
$$

\subsection{Another proof of the existence result}

Here we sketch another proof of Theorem 4.1 that does not use Proposition 4.2. The idea is to use Fedosov's construction with $\Omega=\omega+O(\hbar) \in H^{2}(M)[[\hbar]]$ instead of $\omega$.

Consider $M$ as a $C^{\infty}[[\hbar]]$ manifold. That is we take $\mathcal{O}_{M}^{\hbar}=\mathcal{O}_{M}[[\hbar]]$ as structure sheaf on $M\left(\mathcal{O}_{M}\right.$ is the sheaf of $C^{\infty}$ functions on $\left.M\right)$. For any open $U \subset M$ $\Gamma\left(\mathcal{O}_{M}^{h}, U\right)=\Gamma\left(C^{\infty}(M), U\right)[[\hbar]]$ and a coordinate system on $U$ is specified by the images of the coordinate functions $x_{1}, \ldots, x_{2 n}$ under an isomorphism

$$
F: C^{\infty}\left(U_{o}\right)[[\hbar]] \rightarrow \Gamma\left(\mathcal{O}_{M}^{h}, U\right)
$$

for an open $U_{o} \subset \mathbb{R}^{2 n}$. Any composition $f\left(g_{0}+\hbar g_{1}+\ldots\right)$ for some $C^{\infty}$ functions $f, g_{0}, g_{1}, \ldots$ is computed using the Taylor rule $f\left(g_{0}+\hbar g_{1}+\ldots\right)=\sum_{l=0}^{\infty} f^{(l)}\left(g_{0}\right) G^{l} / l$, $G=\hbar g_{1}+\hbar^{2} g_{2}+\ldots$ One can consider $(M, \Omega)$ as a symplectic $C^{\infty}[[\hbar]]$ manifold and prove the existence of a covering of it with action-angle charts. Let $\pi: M \rightarrow B$ be a fiber bundle which is Lagrangian with respect to $\Omega$ in the usual sense. Using Lemma 4.3 one constructs a symplectic connection $\bar{\Gamma}$ on $(M, \Omega)$ with covariant derivative $\bar{\partial}: \Gamma(T M) \rightarrow \Gamma(T M)[[\hbar]]$, that satisfies properties (3.9)-(3.12) for the data $M, \pi$, $\Omega$. It is obvious that the constant term of $\bar{\partial}$

$$
\partial: T M \rightarrow T M
$$

is a connection with the properties of Theorem 3.1 for the data $M, \pi, \omega$.

Now we can run Fedosov's construction with $\omega$ and $\partial$ replaced by $\Omega$ and $\bar{\partial}$. $W_{M, \hbar}$ will be equiped with the Moyal-Weyl product corresponding to $\Omega$, let us denote it by $\bar{o}$. Consider a Fedosov's connection for $\left(W_{M, \hbar}, \bar{o}\right)$

$$
\bar{D}=\bar{\partial}-\delta+\frac{1}{\hbar} \operatorname{ad}(\bar{\gamma}) .
$$

with curvature form (same) $\Omega$. As in the proof of Theorem 4.4 (see also Lemma 4.5) one proves that it induces a star product $*$ on $M$ which keeps $\pi^{*}\left(C^{\infty}(B)\right)$ 
undeformed. In the remaining part of this Remark we show that $*$ has characteristic class $[\Omega]$.

One can find a fiberwise linear map $F: T M \rightarrow T M[[\hbar]]$, such that $F(\omega)=\Omega$. Then

$$
F^{*}:\left(W_{M, \hbar}, \bar{\circ}\right) \rightarrow\left(W_{M, \hbar}, \circ\right)
$$

is an isomorphism. Continue $F^{*}$ to $W_{M, \hbar} \otimes \Lambda$ trivialy on the second factor. Clearly

$$
\begin{aligned}
& F^{*} \bar{\partial}\left(F^{*}\right)^{-1}=\partial+\operatorname{ad}_{\circ}(\alpha) \text { and } \\
& F^{*} \delta\left(F^{*}\right)^{-1}=\delta+\operatorname{ad}_{\circ}(\beta)
\end{aligned}
$$

for some one-forms $\alpha$ and $\beta$ with values in $S^{2}\left(T^{*} M\right)$ and $S^{1}\left(T^{*} M\right)$ respectively. Here $\mathrm{ad}$. refers to the the Lie bracket associated with the product $\bullet$. As a consequence of this we get that

$$
\begin{aligned}
D & :=\left(F^{*}\right)^{-1} \bar{D} F^{*} \\
& =\partial-\delta+\frac{1}{\hbar} \operatorname{ad}_{\circ}\left(F^{*}(\bar{\gamma})+\hbar \alpha-\hbar \beta\right) .
\end{aligned}
$$

is a Fedosov's connection for $\left(W_{M, \hbar}, \circ\right)$. By straightforward computation one shows that the Weyl curvature of $D$ is $\Omega$ and $F^{*}$ induces isomorphism between $W_{\bar{D}}$ and $W_{D}$. It is now clear that the star products coming from $\bar{D}$ and $D$ are equivalent and have characteristic class $[\Omega]$.

\section{Classification of star products preserving a Lagrangian fiber bundle}

The results of this Section are summarized in the following theorem.

Theorem 5.1 Let $*$ be a star product on the symplectic manifold $(M, \omega)$ for which $\pi^{*}\left(C^{\infty}(B)\right)$ generates a commutative subalgebra of $C^{\infty}(M)[[\hbar]]$. Then its characteristic class has a representative $\Omega \in Z^{2}(M)[[\hbar]]$ such that $\pi: M \rightarrow B$ is Lagrangian with respect to $\Omega$ :

$$
\left.\Omega\right|_{\pi^{-1}(b)}=0, \quad \forall b \in B .
$$

In addition, there is always a Fedosov's star product on $(M, \omega)$ that is equivalent to * and keeps $\pi^{*}\left(C^{\infty}(B)\right)$ undeformed.

The first step towards our proof of Theorem 5.1 is a version of a well known fact, which states that any commutative deformation of the algebra of functions on a smooth manifold is equivalent to a trivial one.

Lemma 5.2 Let $*$ be a star product on $(M, \omega)$ for which the algebra generated by $\pi^{*}\left(C^{\infty}(B)\right)$ is commutative. Then there exists an equivalent product $\star$ on $(M, \omega)$ that keeps $\pi^{*}\left(C^{\infty}(B)\right)$ undeformed. 
We will skip the proof of this lemma as it is similar to the proof of Proposition 5.4 that follows next.

The second step in our proof of Theorem 5.1 is to exponentiate $\star$ to a fiberwise star product $\bullet$ on $W_{\hbar}$ using Emmrich-Weinstein construction [7] reviewed in Subsect. 2.5.1 with a symplectic connection $\partial$ having the properties of Theorem 3.1.

Lemma 5.3 Let $\star$ be a star product on $(M, \omega)$ that keeps $\pi^{*}\left(C^{\infty}(B)\right)$ undeformed. Denote the fiberwise star product $\bullet$ on $W_{M, \hbar}$ obtained from $\star$ by the exponentiation construction of Subsect. 2.5.1 using a symplectic connection $\partial$ with the properties of Theorem 3.1 (see eq. (2.33)). Then $\bullet$ keeps each fiber of $W_{\pi, \hbar}$ undeformed.

Proof. In the exponentiation procedure from Subsect. 2.5.1 one first computes the one-form $\gamma$ for the nonlinear flat connection $D^{0}$ on $W$ (see (2.27)). It is done inductively using (2.28). Similarly to the proof of Theorem 4.4 (see especially (4.18)) one gets

$$
\gamma \in \Gamma\left(W_{M}\right)^{(1)}
$$

Thus

$$
\exp (f) \in \Gamma\left(W_{M}\right)^{(0)}=\Gamma\left(W_{B}\right) \quad \forall f \in \pi^{*}\left(C^{\infty}(B)\right)
$$

(see eq. (2.31) for the definition of exp). The closure of

$$
\operatorname{span}\left\{\exp (f)_{x} \mid f \in \pi^{*}\left(C^{\infty}(B)\right)\right\}
$$

in $S\left(T_{x}^{*} M\right)$ is $S\left(\left(N_{\pi}^{*}\right)_{x}\right)=\left(W_{\pi}\right)_{x}, \forall x \in M$, which proves the Lemma.

The next step is to study the equivalence between the product $\bullet$ and the MoyalWeyl product $\circ(2.9)$.

Proposition 5.4 There exists a leafwise differential operator $P$ on $T M$ of the type

$$
P=\mathrm{id}+\hbar P_{1}+\hbar^{2} P_{2}+\ldots
$$

for some differential operators $P_{j}$ on $T M$ with coefficients in $W_{M}$ such that

$$
P(a \bullet b)=P(a) \circ P(b), \quad \forall a, b \in(W)_{x}, x \in M
$$

and

$$
P a=a, \quad \forall a \in\left(W_{\pi}\right)_{x} \subset(W)_{x} .
$$

Proof. Denote by $C^{l}$ the bundle over $M$ whose fiber at $x \in M$ consists of (local) Hochschild $l$-cochains for $(W)_{x}=\mathcal{J}_{0}^{\infty}\left(T_{x} M\right)$ (equipped with the standard fiberwise commutative product). Any such cochain is represented by an $l$-differential operator on the tangent space $T_{x} M$.

Let us define

$$
\begin{aligned}
\left(C^{l}\right)^{s}=\left\{B \in C^{l} \mid\right. & B \text { is represented by an } l \text {-differential operator } \\
& \text { of degree }>1 \text { in at lest one component or it is } \\
& \text { of degree } 1 \text { in any component and } A(B)=0\},
\end{aligned}
$$


where $A$ denotes the complete antisymmetrization and

$$
\left(C^{l}\right)^{a}=\left\{B \in C^{l} \mid B \text { is antisymmetric of degree } 1 \text { in each component }\right\} .
$$

Let $\left(C^{l}\right)_{\pi}^{a},\left(C^{l}\right)_{\pi}^{s}$, and $C_{\pi}^{l}$ be the subbundles of $\left(C^{l}\right)^{a},\left(C^{l}\right)^{s}$, and $C^{l}$ consisting of those cochains that vanish if one of their arguments is in $W_{\pi}$. It is clear that

$$
C_{\pi}^{l}=\left(C^{l}\right)_{\pi}^{a} \oplus\left(C^{l}\right)_{\pi}^{s}
$$

By $\delta$ and [.,. .] (in this proof only) we denote the (fiberwise) differential and the (super) Lie bracket in the Hochschild complex $C . \alpha \in \Gamma\left(C^{2}\right)$ will denote the cochain corresponding to the fiberwise Poisson bracket $\{., .\}_{\mathrm{fib}}$ on $W_{M}$. The following statements are well known:

(i) $\delta\left(\left(C^{l}\right)^{s}\right) \subset\left(C^{l+1}\right)^{s}$ and

$$
\ldots \stackrel{\delta}{\rightarrow}\left(C^{l-1}\right)^{s} \stackrel{\delta}{\rightarrow}\left(C^{l}\right)^{s} \stackrel{\delta}{\rightarrow}\left(C^{l+1}\right)^{s} \stackrel{\delta}{\rightarrow} \ldots
$$

is acyclic. In particular

$$
\left(C^{1}\right)^{s} \stackrel{\delta}{\rightarrow}\left(C^{2}\right)^{s}
$$

is an inclusion onto $\operatorname{Ker} \delta_{2}\left(\left(C^{2}\right)^{s} \rightarrow\left(C^{3}\right)^{s}\right)$.

(ii) $(T M,\{.,\}$.$) is a regular Poisson manifold with symplectic leaves T_{x} M, x \in M$. The restriction of $\alpha$ to each leaf canonically identifies the fiber of $\left(C^{l}\right)^{s}$ at $x$ with the space of forms $\Omega\left(T_{x} M\right)$ on $T_{x} M$ with coefficients in $(W)_{x}=\mathcal{J}_{0}^{\infty}\left(T_{x} M\right)$. Under this identification $\{\alpha,$.$\} goes to the fiberwise differential d_{T M}$ (see [15]).

We prove that there exists a differential operator $P$ as in (5.3) satisfying (5.4) such that

$$
P_{n} \in \Gamma\left(\left(C^{2}\right)_{\pi}\right) .
$$

The last equation obviously implies that $P$ also satisfies (5.5). According to (5.8) $P_{n}$ should decompose as

$$
P_{n}=P_{n}^{s}+P_{n}^{a}, \text { where } P_{n}^{s} \in \Gamma\left(\left(C^{1}\right)_{\pi}^{s}\right) \text {. and } P_{n}^{a} \in \Gamma\left(\left(C^{1}\right)_{\pi}^{a}\right)
$$

The operator $P$ is constructed iteratively. At the $n$-th step assuming that (5.4) holds modulo $\hbar^{n-1}$, we define $P_{n}^{a}$ and $P_{n-1}^{s}$ in such a way that (5.4) is true modulo $\hbar^{n}$. The equations for $P_{n}^{s}$ and $P_{n-1}^{a}$ are

$$
\begin{aligned}
& \delta P_{n}^{s}=Q_{n}^{s}, \\
& {\left[\alpha, P_{n-1}^{a}\right]=Q_{n-1}^{a} .}
\end{aligned}
$$

with some $Q_{n}^{s} \in \Gamma\left(\left(C^{2}\right)_{\pi}^{s}\right)$ and $Q_{n-1}^{a} \in \Gamma\left(\left(C^{2}\right)_{\pi}^{a}\right)$, which are known from the previous steps and satisfy

$$
\begin{aligned}
& \delta Q_{n}^{a}=0, \\
& {\left[\alpha, Q_{n-1}^{s}\right]=0 .}
\end{aligned}
$$

According to (i) (5.10) has a unique solution $P_{n}^{s}$ in $\Gamma\left(\left(C^{1}\right)^{s}\right)$ and this solutions is in $\Gamma\left(\left(C^{1}\right)_{\pi}^{s}\right)$. The existence of a solution $P_{n-1}^{a} \in \Gamma\left(\left(C^{1}\right)_{\pi}^{a}\right)$ of $(5.11)$ can be proved 
in the following way. The identification from (ii) associates with $Q_{n-1}^{a}$ a family of two-forms $\beta_{x}^{2}$ on $T_{x} M, x \in M$ with coefficients in $\mathcal{J}_{0}^{\infty}\left(T_{x} M\right)$. The restriction of $\beta_{x}^{2}$ to $T_{x} M^{b}$ vanishes for any $x \in M$ (here $M^{b}$ denotes the fiber of $\pi$ passing trough $x$ ). A formal analog of Lemma 4.3 implies that there exists a family of one forms $\beta_{x}^{1}$ on $T M$ whose resetrictions to $T_{x} M^{b}$ vanish and on each tangent space $T_{x} M$

$$
d \beta_{x}^{1}=\beta_{x}^{2} .
$$

Applying the identification from (ii) back to $\beta_{x}^{1}$ plus some standard gluing arguments give the existence of a solution to (5.11) of the desired form.

Finally Theorem 5.1 follows directly from the next Theorem, which makes it more precise.

Theorem 5.5 Let $*$ be a star product on $(M, \omega)$ for which $\pi^{*}\left(C^{\infty}(B)\right)$ generates a commutative subalgebra in $C^{\infty}(M)[[\hbar]]$. Then there exists an injective homomorphism

$$
\rho:\left(C^{\infty}(M)[[\hbar]], *\right) \hookrightarrow\left(\Gamma\left(W_{M, \hbar}\right), \circ\right)
$$

such that

$$
\rho\left(\pi^{*}\left(C^{\infty}(B)\right)\right) \subset \Gamma\left(W_{B, \hbar}\right) .
$$

There exists a Fedosov's connection $D$ on $W_{\hbar}$ for which

$$
\rho=\sigma_{D}
$$

Its Weyl curvature satisfies (5.1). The corresponding Fedosov's product is equivalent to $*$ and keeps $\pi^{*}\left(C^{\infty}(B)\right)$ undeformed.

Proof. Most of the work needed is already done. The homomorphism $\rho$ is just the composition of the maps from Lemmas 5.2, 5.3, and Proposition 5.4. It has properties (i) and (ii) of Subsect. 2.5.1 and from Theorem 2.7 we get that there exists a Fedosov connection $D=\partial-\delta+(1 / \hbar) \operatorname{ad}(\gamma)$ satisfying (5.16). Then for any $f \in C^{\infty}(M)$

$$
\left(\partial-\delta-\frac{1}{\hbar} \operatorname{ad}(\gamma)\right) \rho(f)=0, \quad \forall f \in C^{\infty}(M) .
$$

We have the freedom to choose the initial connection $\partial$. If it has the properties of Theorem 3.1, then

$$
(\partial-\delta) \rho(f) \in \Gamma\left(W_{M, \hbar} \otimes \Lambda^{1}\right)^{(0)}, \quad \forall f \in \pi^{*}\left(C^{\infty}(B)\right),
$$

and thus

$$
[\gamma, \rho(f)] \in \Gamma\left(W_{M, \hbar} \otimes \Lambda^{1}\right)^{(0)}, \quad \forall f \in \pi^{*}\left(C^{\infty}(B)\right) .
$$

The operator $P$ from Proposition 5.4 acts trivially on $\pi^{*}\left(C^{\infty}(B)\right)$, so

$$
\rho(f)=\exp (f) \in \Gamma\left(W_{\pi, \hbar}\right), \quad \forall f \in \pi^{*}\left(C^{\infty}(B)\right),
$$

where exp is the exponential map from the proof of Lemma 5.3. This proves the fact about Fedosov's product in Theorem 5.5. It also implies that the completion of

$$
\operatorname{span}\left\{\rho(f)_{x} \mid f \in \pi^{*}\left(C^{\infty}(B)\right)[[\hbar]]\right\}
$$


in $\left(W_{\hbar}\right)_{x}$ is $\left(W_{\pi, \hbar}\right)_{x}(\forall x \in M)$ and therefore that for any open domain $U \subset B$

$$
\left.\gamma\right|_{U} \in \Gamma\left(W_{M, \hbar} \otimes \Lambda^{1}, \pi^{-1}(U)\right)^{(1)}+\Gamma\left(W_{\pi, \hbar} \otimes \Lambda^{1}, \pi^{-1}(U)\right) .
$$

It is clear that in local action-angle coordinates $(I, \varphi)$

$$
\left(W_{\pi}\right)_{m}=\mathbb{R}\left[\left[J^{1}, \ldots, J^{n}\right]\right]
$$

and that it is the commutant of itself in

$$
\left(\left(W_{M, \hbar}\right)_{m}=\mathbb{R}\left[\left[J^{1}, \ldots, J^{n}, \psi^{1}, \ldots, \psi^{n}\right]\right], \circ\right) .
$$

Recall that by $J^{l}$ and $\psi^{l}$ we denote the images of $d I^{l}$ and $d \varphi^{l}$ in $(W)_{x}$ respectively, $x \in M$. At the end the Weyl curvature of $D$ is

$$
\begin{aligned}
\Omega & =R+\partial \gamma-\delta \gamma+\frac{1}{\hbar} \gamma^{2} \\
& =(\gamma \circ \gamma)_{0}
\end{aligned}
$$

(see the discussion before Theorem 2.4 for the definition of constant term $(.)_{0}$. ) Locally write $\gamma$ as a sum of two terms according to (5.17). Then nonzero contribution to $(\gamma \circ \gamma)_{0}$ give terms of the following type only:

$$
\begin{aligned}
& \gamma_{1}^{\prime} \circ \gamma_{1}^{\prime \prime}, \text { for } \gamma_{1}^{\prime}, \gamma_{1}^{\prime \prime} \in \Gamma\left(W_{M, \hbar}^{j} \otimes \Lambda^{1}\right)^{(1)}, j=1 \text { or } 2, \\
& \gamma_{1} \circ \gamma_{2}, \text { for } \gamma_{1} \in \Gamma\left(W_{M, \hbar}^{1} \otimes \Lambda^{1}\right)^{(1)}, \gamma_{2} \in \Gamma\left(W_{\pi, \hbar} \otimes \Lambda^{1}\right) .
\end{aligned}
$$

In case (5.19)

$$
\gamma_{1}^{\prime} \circ \gamma_{1}^{\prime \prime} \in \Gamma\left(\Lambda^{2}\right)^{(2)}[[\hbar]]
$$

and all forms in $\Gamma\left(\Lambda^{2}\right)^{(2)}[[\hbar]]$ satisfy (5.1).

Consider case (5.20). Locally $\gamma_{1}$ is a polynomial in $\psi^{j}$ of degree at most 1 . The product $(5.20)$ eats out the $\psi$ 's and what remains is in $\Gamma\left(\Lambda, \pi^{-1}(U)\right)^{(0)}[[\hbar]]$. As a one-form it vanishes on $M^{b}, \forall b \in B$ and so does $\gamma_{1} \circ \gamma_{2}$. This ends the proof of Theorem 5.5.

\section{References}

[1] Arnold, V.: Mathematical methods of classical mechanics. Graduate texts in mathematics Vol. 60, Springer, Berlin, 1978

[2] Berezin, F. A.: Quantization. Math. USSR Izv. 8, 1109-1165 (1974)

[3] Bayen, F., Flato, .M., Fronsdal C., Lichnerowicz, A., and Sternheimer, D.: Deformation theory and quantization, I and II. Ann. Phys. 111, 61-151 (1977)

[4] Deligne, P.: Déformations de l'algèbre des fonctions d'une variété symplectique: Comparaison entre Fedosov et De Wilde, Lecomte. Selecta Math. (N.S.) 1, 667-697 (1995) 
[5] Duistermaat, J., J.: On global action-angle coordinates. Commun. Pure Appl. Math. XXXIII, 687-706 (1980)

[6] De Wilde, M., and Lecomte, P. B. A.: Existence of star-products and of formal deformations of the Poisson Lie algebra of arbitrary symplectic manifolds. Lett. Math. Phys. 7, 487-496 (1983)

[7] Emmrich, C., and Weinstein, A.: The differential geometry of Fedosov's construction. In: Lie theory and geometry in honor of B. Kostant, Progress in Math 123, Birkhauser, New York, 1994

[8] Fedosov, B.: A simple geometric construction of deformation quantization. J. Diff. Geom. 40, 213-238 (1994)

[9] Fedosov, B.: Deformation quantization and index theory. In: Mathematical topics 9, Akademie Verlag, Berlin, 1996

[10] Gerstenhaber, M.: On the deformation of rings and algebras, I. Ann. Math. 79, 59-103 (1964)

[11] Guillemin, V., and Sternberg, S.: Symplectic techniques in physics. Cambridge University Press, Cambridge (1984)

[12] Kontsevich, M.: Deformation quantization of Poisson manifolds, I. preprint math/9709180 (1997)

[13] Moser, J.: On the volume elements on a manifold. Trans. AMS 120, 280296 (1965)

[14] Moyal, J.: Quantum mechanics as a statistical theory. Proc. Camb. Phil. Soc. 45, 99-124 (1949)

[15] Nest, R., and Tsygan, B.: Algebraic index theorem for families. Adv. Math. 113, 151-205 (1995)

[16] Vey, J.: Déformations du crochet de Poisson sur une variété symplectique. Comment. Math. Helv. 50, 421-454 (1975)

[17] Weinstein, A.: Deformation quantization. Séminaire Bourbaki, 46ème année, N. 789 (1993-1994), Asterisque 227, 389-409 (1995)

[18] Weyl, H.: Quantenmechanik und Gruppentheorie. Z. Phys. 46, 1-46 (1927)

[19] Xu, P.: Fedosov's *-products and quantum momentum maps. Comm. Math. Phys. 197, 167-197 (1998) 